\title{
On the existence of fractal strings whose set of dimensions of fractality is not perfect
}

\author{
G. Mora • J.M. Sepulcre • T. Vidal
}

Received: date / Accepted: date

\begin{abstract}
In this paper we give an example of a nonlattice self-similar fractal string such that the set of real parts of their complex dimensions has an isolated point. This proves that, in general, the set of dimensions of fractality of a fractal string is not a perfect set.
\end{abstract}

Keywords Nonlattice Dirichlet polynomials · Fractal strings · Nonlattice self-similar fractal strings.

Mathematics Subject Classification (2000) 30D20 $28 \mathrm{~A} 80$

\section{Introduction}

Lapidus and van Frankenhuysen (see, for example, [1, p. 42]) define a fractal string $\mathcal{L}$ as a disjoint union of open intervals, the lengths of which form a sequence $\mathcal{L}=l_{1}, l_{2}, l_{3}, \ldots$, of finite length $|\mathcal{L}|=\sum_{j=1}^{\infty} l_{j}$. The geometric zeta function of $\mathcal{L}$, denoted by $\zeta_{\mathcal{L}}(s)$, is defined as

$$
\zeta_{\mathcal{L}}(s):=\sum_{j=1}^{\infty} l_{j}^{s}, \quad s=\sigma+i t
$$

and, under the assumption that it has a meromorphic continuation (also denoted by $\left.\zeta_{\mathcal{L}}(s)\right)$ to the left of $D$, the abcissa of convergence of $\zeta_{\mathcal{L}}(s)$, its poles are called the complex dimensions of $\mathcal{L}$.

The mentioned authors, in [1, p. 55], introduce the class of self-similar fractal strings, and they prove (see $[1$, Theorem 5.2]) that the geometric zeta

Preprint submitted to RACSAM, 2014.

G. Mora · J.M. Sepulcre · T. Vidal

Department of Mathematical Analysis

University of Alicante

03080-Alicante, Spain

E-mail: gaspar.mora@ua.es, JM.Sepulcre@ua.es, tmvg@alu.ua.es 
function of each string of the above class has a meromorphic continuation to the whole complex plane, given by

$$
\zeta_{\mathcal{L}}(s)=\frac{L^{s} \sum_{k=1}^{K} g_{k}^{s}}{1-\sum_{j=1}^{N} r_{j}^{s}}, \quad \text { for } s \in \mathbb{C} .
$$

Here, $L=\zeta_{\mathcal{L}}(1)$ is the total length of $\mathcal{L}$, the $r_{j}$ 's $(N \geq 2)$, called scaling ratios, are real numbers of the interval $(0,1)$ satisfying $\sum_{j=1}^{N} r_{j}<1$, with

$$
1>r_{1} \geq r_{2} \geq \ldots \geq r_{N}>0
$$

and the $g_{k}$ 's $(K \geq 1)$, called gaps, are positive real numbers such that

$$
\sum_{j=1}^{N} r_{j}+\sum_{k=1}^{K} g_{k}=1
$$

Whenever the Dirichlet polynomial,

$$
P(s)=1-\sum_{j=1}^{N} r_{j}^{s}
$$

has repeated scaling ratios, (1.3) becomes

$$
P(s)=1-\sum_{j=1}^{M} m_{j} r_{j}^{s},
$$

where the $m_{j}$ 's, called multiplicities, are positive integers. Then, the distinct scaling ratios $1>r_{1}>\ldots>r_{M}>0(M \geq 2)$, jointly with their multiplicities, satisfy the condition

$$
\sum_{j=1}^{M} m_{j} r_{j}<1 .
$$

Therefore, each self-similar fractal string $\mathcal{L}$ has an associated Dirichlet polynomial $P(s)$ of the form (1.4) satisfying (1.5). Then, according to the definition of the set $\mathcal{D}_{\mathcal{L}}$ of complex dimensions of $\mathcal{L}$, from (1.1), one has that $\mathcal{D}_{\mathcal{L}} \subset Z_{P(s)}$, the set of zeros of $P(s)$. The authors introduce the nonlattice case (see, for instance, $[3$, p. 65]) when

$$
\frac{\log r_{j}}{\log r_{1}} \notin \mathbb{Q}, \quad \text { for some } j \geq 2,
$$

which means that at least $\log r_{1}$ and $\log r_{j}$, for some $j \geq 2$, are linearly independent over the rationals. Whether all numbers in the set $\left\{\log r_{j}: 1 \leq j \leq M\right\}$ are linearly independent over the rationals, Lapidus and van Frankenhuysen say that this is the generic nonlattice case. The nonlattice and generic nonlattice terms are assigned to both fractal strings and their associated Dirichlet polynomials. 
In the present paper we are going to prove that the nonlattice condition (1.6) is not sufficient to assure that the set of the real parts of the complex dimensions of a fractal string (or equivalently the set of the real parts of the zeros of its associated nonlattice Dirichlet polynomial) does not have isolated points. Even the much more restrictive class of self-similar fractal strings contains, at least, an element such that the set of real parts of its complex dimensions has an isolated point.

\section{The counterexample}

Theorem 1 There exist nonlattice self-similar strings for which the set of real parts of their complex dimensions has isolated points.

Proof Consider the fractal string $\mathcal{L}$ defined by the three scaling ratios $r_{1}=$ $1 / 27, r_{2}=1 / 64, r_{3}=1 / 216$ of multiplicities $m_{1}=3, m_{2}=1, m_{3}=3$, and a gap $g_{1}=55 / 64$. Then we have

$$
\sum_{j=1}^{3} m_{j} r_{j}+g_{1}=\frac{9}{64}+\frac{55}{64}=1
$$

so (1.2) is fulfilled and, consequently, $\mathcal{L}$ is a self-similar fractal string. On the other hand as

$$
\frac{\log r_{2}}{\log r_{1}}=\frac{2 \log 2}{\log 3} \notin \mathbb{Q},
$$

$\mathcal{L}$ is a nonlattice string. Moreover, noting (1.1), the set of complex dimensions of $\mathcal{L}$ coincides with the set of zeros of its Dirichlet polynomial associated

$$
P(s)=1-3 \frac{1}{27^{s}}-\frac{1}{64^{s}}-3 \frac{1}{216^{s}}, \quad s=\sigma+i t .
$$

Now we claim that the set of real parts of the zeros of $P(s)$ has an isolated point. Indeed, let $R_{P(s)}$ be the set of real parts of the zeros of $P(s)$. Consider a complex number $s$ such that $8^{-s}=-1$. Necessarily, $s$ is of the form

$$
s_{k}=\frac{(2 k+1) \pi i}{3 \log 2}, \quad k \in \mathbb{Z} .
$$

Then each $s_{k}$ satisfies

$$
P\left(s_{k}\right)=1-3 \frac{1}{27^{s_{k}}}-\frac{1}{8^{s_{k}} 8^{s_{k}}}-3 \frac{1}{27^{s_{k}} 8^{s_{k}}}=1-3 \frac{1}{27^{s_{k}}}-1+3 \frac{1}{27^{s_{k}}}=0 .
$$

Thus, $s_{k}$ is a zero of $P(s)$ and, taking into account (2.1), its real part, 0 , belongs to $R_{P(s)}$. Now we say that 0 is an isolated point of $R_{P(s)}$. If this is not so, there exists a sequence of zeros of $P(s)$, say $\left(w_{n}\right)_{n \geq 1}$, with $\sigma_{n}:=\operatorname{Re} w_{n} \neq 0$ such that

$$
\lim _{n \rightarrow \infty} \sigma_{n}=0
$$


Since $\sigma_{n} \neq 0$, it follows that $8^{-w_{n}} \neq-1$ for all $n \geq 1$. It is immediate that

$$
\frac{P\left(w_{n}\right)}{8^{-w_{n}}+1}=1-3 \frac{1}{27^{w_{n}}}-\frac{1}{8^{w_{n}}}=0
$$

because $P\left(w_{n}\right)=0$. Therefore,

$$
1=3 \frac{1}{27^{w_{n}}}+\frac{1}{8^{w_{n}}},
$$

and by taking moduli we get

$$
1=\left|3 \frac{1}{27^{w_{n}}}+\frac{1}{8^{w_{n}}}\right| \geq 3 \frac{1}{27^{\sigma_{n}}}-\frac{1}{8^{\sigma_{n}}}, \quad \text { for all } n \geq 1 .
$$

Finally, by taking the limit and appealing to (2.2), we are led to the contradiction that $1 \geq 2$. Hence, as claimed, 0 is an isolated point of $R_{P(s)}$ and our theorem follows.

Finally, in [1, Remark 8.2], one defines the set of dimensions of fractality of a fractal string as the closure of the set of real parts of its complex dimensions. As an easy consequence of the above theorem we obtain the following result.

Corollary 1 There exists a nonlattice self-similar fractal string such that its set of dimensions of fractality is not perfect.

Nevertheless, in [1, Theorem 8.1], [2, Theorem 3.53] and [3, Theorem 3.53] one states the following result:

Theorem 2 (Lapidus and van Frankenhuysen, 2003, 2006 and 2013) The set of real parts of the complex dimensions of a nonlattice string has no isolated points.

\section{References}

1. Lapidus, M.L. and Van Frankenhuysen, M.: Complex Dimensions of Self-Similar Fractal Strings and Diophantine Approximation, Experiment. Math. No. 1, 12, 41-69 (2003)

2. Lapidus, M.L. and Van Frankenhuysen, M.: Fractal geometry, complex dimensions and zeta functions: Geometry and Spectra of Fractal Strings. Springer Monographs in Mathematics. Springer, New York (2006)

3. Lapidus, M.L. and Van Frankenhuysen, M.: Fractal geometry, complex dimensions and zeta functions: Geometry and Spectra of Fractal Strings. Springer Monographs in Mathematics. Second edition. Springer, New York (2013) 